\title{
Patient Expectation Is the Most Important Predictor of Discharge Destination After Primary Total Joint Arthroplasty
}

\author{
Mohamad J. Halawi, MD ${ }^{\mathrm{a}}$, Tyler J. Vovos, BS ${ }^{\mathrm{b}}$, Cindy L. Green, PhD ${ }^{\mathrm{c}}$, Samuel S. Wellman, MD ${ }^{\mathrm{a}}$, \\ David E. Attarian, MD ${ }^{\mathrm{a}}$, Michael P. Bolognesi, MD ${ }^{\mathrm{a}}$ \\ a Department of Orthopaedic Surgery, Duke University Medical Center, Durham, North Carolina \\ b Duke University School of Medicine, Durham, North Carolina \\ c Department of Biostatistics and Bioinformatics, Duke University Medical Center, Durham, North Carolina
}

\section{A R T I C L E I N F O}

\section{Article history:}

Received 20 September 2014

Accepted 23 October 2014

\section{Keywords:}

hip

knee

arthroplasty

discharge destination

preoperative predictors

patient expectation

\begin{abstract}
A B S T R A C T
The purpose of this study was to identify preoperative predictors of discharge destination after total joint arthroplasty. A retrospective study of three hundred and seventy-two consecutive patients who underwent primary total hip and knee arthroplasty was performed. The mean length of stay was 2.9 days and $29.0 \%$ of patients were discharged to extended care facilities. Age, caregiver support at home, and patient expectation of discharge destination were the only significant multivariable predictors regardless of the type of surgery (total knee versus total hip arthroplasty). Among those variables, patient expectation was the most important predictor $(P<0.001$; OR 169.53). The study was adequately powered to analyze the variables in the multivariable logistic regression model, which had a high concordance index of 0.969 .
\end{abstract}

(c) 2014 Elsevier Inc. All rights reserved.

\section{Introduction}

By 2020, the projected demand for primary total hip and knee arthroplasty in the United States will exceed 1.8 million procedures, a nearly three-fold increase from 2010 [1]. In contrast, the number of hospital beds has steadily decreased nationwide since 1975 [2]. To meet the growing demand for total joint arthroplasty (TJA), patients are now discharged faster and at higher rates to extended care facilities (ECF), such as acute rehabilitation and skilled nursing centers. For example, among Medicare beneficiaries, the mean hospital length of stay (LOS) after total hip arthroplasty (THA) has decreased from 9.1 days to 3.1 days between 1991 and 2008 with a concomitant four-fold increase in the rate of discharge to ECF [3].

Health economics studies suggest that the downward trend in hospital beds is only one factor driving the shift of postoperative rehabilitation burden to ECF. Shortages of nursing staff [4] and financial incentives related to Medicare's prospective payment system (PPS) are also contributing factors [5]. Implemented in 1983, PPS provides fixed payments to hospitals per discharge based on illness categories known as diagnosis related groups. Multiple previous investigators have demonstrated increased rates of discharge to skilled nursing facilities (SNFs) following the implementation of PPS [6-8].

The Conflict of Interest statement associated with this article can be found at http:// dx.doi.org/10.1016/j.arth.2014.10.031.

Reprint requests: Mohamad J. Halawi, MD, Department of Orthopaedic Surgery, Duke University Medical Center Box 3000, Durham, North Carolina 27710.
The current health care climate of capacity and staffing shortages implores the need for increased understanding of factors influencing discharge destination in order to ensure operational efficiency, timely discharge, shorter LOS, cost-effective care, and higher patient satisfaction. Most importantly, understanding the factors associated with discharge destination may help increase the rates of discharge to home as opposed to ECF. Building on previous findings, the aim of this study was to identify readily available factors that can be used for preoperative prediction of discharge destination. To our knowledge, this is the first study to examine predictors of discharge in an exclusively primary TJA patient population reflecting current national trends with regards to LOS and rate of discharge to ECF. Most importantly, this is the first study to critically assess the effects of factors such as preoperative pain level and patient expectation on discharge destination.

\section{Material and Methods}

IRB approval was obtained. The primary outcome measure of the study was discharge destination. All patients admitted to our institution between January 1, 2012 and December 31, 2012 with CPT codes 27447 (total knee arthroplasty) and 27130 (total hip arthroplasty) as the primary procedures were included in the study. Three hundred and seventy-two patients were identified and their medical records were retrospectively reviewed. Three surgeons performed the operations using a variety of cementless THA and cemented TKA implants.

All patients were admitted from home on the day of surgery. Unless contraindicated, spinal anesthesia was performed on a routine basis. In 
addition, patients undergoing THA received single shot fascia iliaca block and those undergoing TKA received continuous femoral nerve block. Following surgery, patients had a standardized clinical pathway with regards to pain control, mobilization, and anticoagulation. In general, intravenous patient-controlled analgesia was provided immediately following surgery and discontinued on the first postoperative day. Physical and occupational therapy (PT/OT) was initiated on the day of surgery and continued daily until discharge. The primary goals of the first PT/OT session were to assess patients' needs, review acute rehabilitation goals, assist with mobility as tolerated, and initiate preliminary discharge planning. As part of the preliminary discharge planning, the therapists inquired about patients' expectation of discharge destination, which was grouped into either home or extended care facility (acute rehabilitation and skilled nursing care). During the period in which the joint arthroplasties in this study were performed, patients were provided with informational packets in clinic describing the procedures, benefits, complications, and rehabilitation goals. They were also given the option of attending a total joint camp, but participation was less than $20 \%$. However, patients were informed by their surgeons that the decision for discharge to home vs. ECF primarily depended on their progress with PT/OT.

Unless contraindicated, enoxaparin was used for DVT prophylaxis. Patients were cleared for discharge when they were medically stable, had adequate pain control, were able to void and tolerate oral diet, had no surgical concerns, and were functionally suitable for their discharge destination as determined by PT/OT.

The variables analyzed were age, gender, body mass index (BMI), number of comorbidities, history of TJA, history of admission to ECF, pain level, use of assistive device, procedure laterality (unilateral vs. bilateral), caregiver assistance at home, type of surgery (THA vs. TKA), and patient expectation of discharge destination. A comorbid condition was defined as any condition (medical or mental) that was actively treated at the time of surgery. Because a majority of patients carried a diagnosis of arthritis and were receiving medications for joint pain, arthritis was counted as a comorbid condition only if it involved joints other than the replaced knee(s). We intentionally chose to assess health status based on the number of active conditions in order to eliminate the use of scoring systems, which was one of the goals of this study. There is currently no gold-standard instrument for evaluating comorbidities; and even the most widely used scoring systems (American Society of Anesthesiologists score and Charlson comorbidity index) are not allinclusive or have moderate inter-rater reliability [9-11]. For example, while the Charlson comorbidity index, initially designed as a predictor for mortality [12], is an excellent prognostic tool in life-threatening conditions [13], it may not be sensitive enough for low-mortality elective procedures [14] and it does not account for conditions that may impact the postoperative course, such as depression, anxiety, fibromyalgia, and back pain. Caregiver assistance was classified as either adequate for patient needs (meals, hygiene, etc.) or inadequate. Pain level was assessed at the time of preoperative anesthesia evaluation and collected using the numeric rating scale for pain. Patient expectation of discharge destination was collected at the time of initial PT/OT session as described above.

Three hundred and seventy-two patients were included in this study. There were 170 males and 202 females with a mean age of 63.3 years (range 25-92). The mean BMI, pain level, and number of comorbidities were 31.5 (range 17.0-50.8), 3.9 (range 0.0-10.0), and 2.8 (range 0.0-9.0) respectively. All patients were admitted from home and only 5 (1.3\%) patients had history of admission to ECF. 130 patients (34.9\%) used assistive devices. 8 patients $(2.2 \%)$ underwent bilateral surgery. 103 patients (27.7\%) had history of at least one lower extremity total joint arthroplasty. 319 patients $(85.8 \%)$ had adequate caregiver assistance at home. The demographic features of the study cohort are outlined in Table 1.

Results were summarized using the mean and standard deviation for continuous variables and counts and percentages for categorical
Table 1

Demographic Features of the Study Group ( $n=372$ ).

\begin{tabular}{lcc}
\hline Feature & Home & ECF \\
\hline $\mathrm{N}$ & $264(71.0 \%)$ & $108(29.0 \%)$ \\
Age, years & $61.0 \pm 9.7$ & $68.8 \pm 9.7$ \\
Male gender & $139(52.6 \%)$ & $31(28.7 \%)$ \\
Female gender & $125(47.4 \%)$ & $77(71.2 \%)$ \\
BMI & $31.1 \pm 6.0$ & $32.5 \pm 7.1$ \\
Pain level & $3.8 \pm 3.0$ & $4.1 \pm 3.2$ \\
Number of comorbidities & $2.5 \pm 1.7$ & $3.4 \pm 2.1$ \\
THA surgery & $92(34.8 \%)$ & $20(18.5 \%)$ \\
TKA surgery & $172(65.2 \%)$ & $88(81.5 \%)$ \\
Use of assistive device & $71(26.9 \%)$ & $59(54.6 \%)$ \\
Bilateral surgery & $1(0.4 \%)$ & $7(6.5 \%)$ \\
History of ECF admission & $0(0.0 \%)$ & $5(5.2 \%)$ \\
History of THA & $37(14.0 \%)$ & $9(8.3 \%)$ \\
History of TKA & $37(14.0 \%)$ & $28(25.9 \%)$ \\
Available assistance at home & $258(97.7 \%)$ & $61(56.5 \%)$ \\
Length of stay, days & $2.6 \pm 0.7$ & $3.5 \pm 0.9$ \\
Expectation of discharge to home & $255(96.6 \%)$ & $14(13.3 \%)$ \\
\hline
\end{tabular}

$\mathrm{ECF}=$ extended care facility.

variables. The relationship between each categorical variable and discharge destination was assessed using the chi-square test. If the expected number in a cell was small, Fisher's exact test was used. Analysis of continuous variables was performed using Student's t-test. All statistical tests were two-sided, and a $P$ value $\leq 0.05$ was considered to be statistically significant. Following univariable analyses, significant factors were subjected to a multivariable logistic regression analysis to determine significant variables associated discharge destination. The linearity assumption between each continuous variable and the logit of the probability discharge to extended care facilities was verified and transformations made if necessary. Type of surgery was forced into the final model given the possible inherent confounders that exist between these two groups. Concordance index ( $\mathrm{C}$-index) was used to determine how well the model discriminated between different responses. The conditional power of the logistic regression model was determined according to the sample size, prevalence, and the corresponding odds ratio of significant multivariable model covariates as previously described [15]. Given our sample size was 372 with 108 (40.9\%) being discharged to ECF, we had more than $80 \%$ power (type II error $<0.2$ ) to detect an effect size (delta) of 0.3 using a statistical significance level of 0.05 (type I error $<0.05)$. SAS version 9.2 was used for all analyses.

\section{Results}

The patient characteristics are described in Table 1 . Two hundred and sixty-four patients (71.0\%) were discharged to home and 108 (29\%) were discharged to ECF. The mean LOS was 2.6 days (range 2.0-6.0) for patients discharged to home and 3.5 days (range 2.0-7.0) for patients discharged to ECF. Factors that were not significantly correlated with discharge destination were pain level $(P=0.364)$ and history of THA ( $P=0.131)$. Gender, number of comorbidities, BMI, history of TKA, history of admission to ECF, use of assistive devices, bilateral surgery, and type of surgery (TKA or THA) were only statistically significant in univariable analyses $(P \leq 0.001,0.001,0.044,0.006,0.002,0.001$, 0.001 , and 0.002 , respectively). Age, assistance at home, and patient expectation of discharge destination were the only statistically significant variables after both univariable and multivariable analyses $(P \leq 0.008$, 0.046 , and 0.001 respectively). The $C$-index for the multivariable logistic regression model was 0.969 . The type of surgery (total knee versus total hip arthroplasty; $P=0.15$ ) was left in the final model but was not a significant predictor of discharge destination. The multivariable model had more than $90 \%$ power to detect a significant difference in each of the multivariable final model predictors. Tables 2 and 3 outline both the univariable and multivariable relationships of each preoperative factor with discharge destination. Spline transformations were not necessary to meet the linearity assumptions of the multivariable model, however, 
Table 2

Univariable Analyses of the Influence of Various Factors on Discharge Destination.

\begin{tabular}{lr}
\hline Factor & $P$ Value \\
\hline Age & $<0.001$ \\
Gender & $<0.001$ \\
BMI & 0.044 \\
Pain level & 0.364 \\
Surgery type (THA vs. TKA) & 0.002 \\
Number of comorbidities & $<0.001$ \\
History of THA & 0.131 \\
History of TKA & 0.006 \\
History of ECF admission & 0.002 \\
Use of assistive device & $<0.001$ \\
Caregiver assistance & $<0.001$ \\
Expectation of discharge destination & $<0.001$ \\
Bilateral surgery & $<0.001$ \\
\hline
\end{tabular}

$\mathrm{ECF}=$ extended care facility

age $>60$ represented a significant cut point with regards to increased likelihood of discharge to an ECF $(P<0.001$; Fig. 1$)$.

Twenty-seven patients (7.3\%) developed minor postoperative complications during hospitalization. There was a significant association between discharge to ECF and the development of a complication $(P<$ $0.001)$. Patients discharged to ECF also had significant risk for blood transfusion $(P=0.006)$ and longer $\operatorname{LOS}(P<0.001)$.

\section{Discussion}

As the demand for TJA continues to grow, understanding the factors associated with discharge destination may allow not only efficient and cost-effective operations, but also increased discharge rates to home and improved patient satisfaction. In this study, we showed that age, caregiver assistance, and expectation of discharge destination were significant multivariable preoperative predictors of discharge destination after primary total hip and knee arthroplasty. Among these factors, patient expectation of discharge destination was the most heavily weighted factor. To our knowledge, only one previous study reported such an association. Oldmeadow et al developed the Risk Assessment and Predictor Tool (RAPT) for total joint arthroplasty [16]. Despite demonstrating statistical significance, the authors removed patient expectation of discharge destination from the RAPT prediction system because it was a "potentially unstable" factor. While the RAPT was internally validated by the original authors, other studies found poor concordance with discharge disposition [17]. This is perhaps because RAPT was based on a patient population that had a relatively high mean LOS ( $>9$ days) and included hemiarthroplasty and revision procedures.

Barsoum et al developed a Predicting Location after Arthroplasty Nomogram (PLAN) based on 17 variables, of which only 7 were statistically significant: type of procedure, age, gender, heart disease, diabetes, COPD, and caregiver assistance [17]. The study had a relatively long mean LOS, high rate of discharge to ECF, and a wide variation in weight bearing status as nearly half of their patient sample comprised revision procedures. In contrast, we investigated only patients who had primary TJA and included all active comorbidities. However, like Barsoum et al, we did not find a correlation between BMI and discharge destination, possibly due to the high prevalence of obesity in our patient population (mean BMI 31.5) and that of Barsoum et al (mean BMI 31.4). Obesity has

Table 3

Multivariable Logistic Regression Model for Preoperative Predictors of Discharge to Extended Care Facilities (ECF).

\begin{tabular}{lrc}
\hline Factor & $P$ Value & Odds Ratio (95\% Confidence Interval) \\
\hline Age & 0.008 & $1.10(1.02-1.18)$ \\
Inadequate caregiver assistance & 0.046 & $4.43(1.02-19.18)$ \\
Expectation of discharge to ECF & $<0.001$ & $169.53(60.67-473.76)$ \\
Surgery type (TKA) & 0.145 & $2.45(0.73-8.26)$ \\
\hline
\end{tabular}

Estimated Spline Transformation and 95\% C.I.

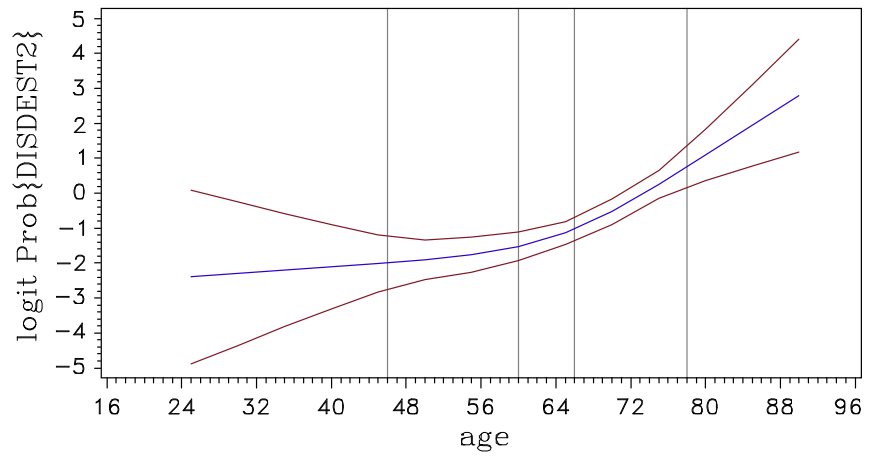

Fig. 1. Age $>60$ represented a significant cut-off point for increased likelihood of discharge to extended care facilities.

particular implications in patients undergoing joint arthroplasty with studies showing a positive correlation with poor preoperative health [18] and high postoperative complications [19]. While obesity was not a significant predictor in our study and our rates of discharge to ECF remained consistent with national data despite high prevalence of obesity [3], the distinct preoperative characteristics of obese patients undergoing joint arthroplasty (e.g. low WOMAC and SF-36 scores) [18] make them especially suitable for targeted preoperative education and discussion of expectations to minimize LOS and discharges to ECF.

We recognize that the retrospective nature of this study may represent a weakness. However, this study design may also eliminate observer bias as the data were collected without the intent of the study at that time. While our patient sample is small compared to previously published studies that were based on large-scale database searches, we had sufficient statistical power for the significant factors in the multivariable logistic regression model. In addition, the $\mathrm{C}$-index, which is a measure of how well the multivariable logistic regression model discriminated between different responses, was quite robust. We analyzed a consecutive group of patients who underwent primary total hip and knee arthroplasty without exclusion criteria. Patients underwent a standardized postoperative clinical pathway with clear discharge criteria. All the preoperative factors are easily obtainable with no need for scoring systems. By reviewing individual patient charts, we had the advantage of assessing factors that are sometimes not routinely collected in large-scale databases, such as caregiver assistance, patient expectations, and pain level. Furthermore, unlike previous studies, we did not include revision TJA in the study sample as the complexity, variation in postoperative care pathways, and longer LOS could skew the results. Most importantly, our sample reflects national trends with regards to mean LOS and rate of discharge to ECF [3], which makes the results generalizable.

Our study has demonstrated for the first time that patient expectation of discharge destination is the most important predictor of final disposition after primary total joint arthroplasty. We have also confirmed earlier findings, particularly the significance of age and caregiver assistance at home in determining the discharge destination $[17,20]$. While caregiver assistance may not be a modifiable factor, patient expectation represents a compelling target for intervention as it carried the heaviest weight in our prediction model. Patient expectation is a subjective measure of one's mindset and can be influenced by complex variables, such as anxiety, self-confidence, and knowledge. By providing better preoperative education, the surgeon can help manage patient expectations and promote feelings of safety and readiness for discharge $[21,22]$. Targeted postoperative care based on preoperative risk assessment has also been shown to increase the number of patients discharged directly to home [23]. Our group has recently started incorporating the determination of discharge destination in the preoperative clinic visit. This has allowed us to moderate expectations according to risk factors 
early in the process. By adopting this intervention, we have noticed a dramatic decline in both LOS and rates of ECF discharges.

\section{References}

1. Kurtz SM, Ong KL, Lau E, et al. Impact of the economic downturn on total joint replacement demand in the United States: updated projections to 2021. J Bone Joint Surg Am 2014;96:624.

2. National Center for Health Statistics. Hospitals b, and occupancy rates, by type of ownership and size of hospital: United States, selected years 1975-2009; 2011 [Hyattsville, Maryland].

3. Cram P, Lu X, Kaboli PJ, et al. Clinical characteristics and outcomes of Medicare patients undergoing total hip arthroplasty, 1991-2008. JAMA 2011;305:1560.

4. Bazzoli GJ, Brewster LR, Liu G, et al. Does U.S. hospital capacity need to be expanded? Health Aff 2003;22:40.

5. Kahn KL, Rubenstein LV, Draper D, et al. The effects of the DRG-based prospective payment system on quality of care for hospitalized Medicare patients. An introduction to the series. JAMA 1990;264:1953.

6. DesHarnais S, Chesney J, Fleming S. Trends and regional variations in hospital utilization and quality during the first two years of the prospective payment system. Inquiry $1988 ; 25: 374$

7. Guterman S, Eggers PW, Riley G, et al. The first 3 years of Medicare prospective payment: an overview. Health Care Financ Rev 1988;9:67.

8. Fitzgerald JF, Moore PS, Dittus RS. The care of elderly patients with hip fracture. Changes since implementation of the prospective payment system. N Engl J Med 1988;319:1392.

9. Bjorgul K, Novicoff WM, Saleh KJ. Evaluating comorbidities in total hip and knee arthroplasty: available instruments. J Orthop Traumatol 2010;11:203.

10. Sankar A, Johnson SR, Beattie WS, et al. Reliability of the American Society of Anesthesiologists physical status scale in clinical practice. Br J Anaesth 2014;113:424-32.
11. Aronson WL, McAuliffe MS, Miller K. Variability in the American Society of Anesthesiologists Physical Status Classification Scale. AANA J 2003:71:265.

12. Charlson ME, Pompei P, Ales KL, et al. A new method of classifying prognostic comorbidity in longitudinal studies: development and validation. J Chronic Dis 1987;40: 373.

13. Kastner C, Armitage J, Kimble A, et al. The Charlson comorbidity score: a superior comorbidity assessment tool for the prostate cancer multidisciplinary meeting. Prostate Cancer Prostatic Dis 2006;9:270.

14. Harse JD, Holman CD. Charlson's Index was a poor predictor of quality of life outcomes in a study of patients following joint replacement surgery. J Clin Epidemiol 2005; $58: 1142$.

15. Demidenko E. Sample size determination for logistic regression revisited. Stat Med 2007;26:3385.

16. Oldmeadow LB, McBurney H, Robertson VJ. Predicting risk of extended inpatient rehabilitation after hip or knee arthroplasty. J Arthroplast 2003;18:775.

17. Barsoum WK, Murray TG, Klika AK, et al. Predicting patient discharge disposition after total joint arthroplasty in the United States. J Arthroplast 2010;25:885.

18. Vulcano E, Lee YY, Yamany T, et al. Obese patients undergoing total knee arthroplasty have distinct preoperative characteristics: an institutional study of $4718 \mathrm{pa}-$ tients. J Arthroplast 2013;28:1125.

19. Huddleston JI, Wang Y, Uquillas C, et al. Age and obesity are risk factors for adverse events after total hip arthroplasty. Clin Orthop Relat Res 2012;470:490.

20. Bozic KJ, Wagie A, Naessens JM, et al. Predictors of discharge to an inpatient extended care facility after total hip or knee arthroplasty. J Arthroplast 2006; 21(6 Suppl 2):151.

21. Mahomed NN, Koo Seen Lin MJ, Levesque J, et al. Determinants and outcomes of inpatient versus home based rehabilitation following elective hip and knee replacement. J Rheumatol 2000;27:1753.

22. Heine J, Koch S, Goldie P. Patients' experiences of readiness for discharge following a total hip replacement. Aust J Physiother 2004;50:227.

23. Oldmeadow LB, McBurney H, Robertson VJ, et al. Targeted postoperative care improves discharge outcome after hip or knee arthroplasty. Arch Phys Med Rehabil 2004;85:1424 